Z. klin. Chem. u. klin. Biochem.

8. Jg., S. 170-172, März 1970

\title{
Statistische Qualitätskontrollen mit Rinderserum als Untersuchungsmaterial
}

\author{
Von K. Borner, W. Fabricius und B. Marowyski \\ Aus dem Institut für Klinische Chemie und Klinische Biocbemie der Freien Universität Berlin, Klinikum Steglitz \\ (Direktor: Prof. Dr. H.J. Dulce)
}

(Eingegangen am 30. November 1969)

Es wird über die Eignung von Rinderserum zur Kontrolle einer Reihe klinisch-chemischer Routineanalysen berichtet. Die Präparation des Materials, die Parameter der Qualitätskontrolle und die Haltbarkeit von 16 Komponenten des Serums werden beschrieben. Wegen seines niedrigen Preises und fehlender.Hepatitisgefahr wird Rinderserum den käuflichẹn Testseten und menschlichem Sammelserum als Kontrollmaterial vorgezogen.

\section{Statistical quality control with bovine serum as the test material}

The suitability of bovine serum as a control material for a series of routine clinical chemical analyses is reported. The preparation of the material, the parameters of the quality control and the stability of 16 components of the serum are described. Owing to its low price and the absence of a hepatitis risk, bovine serum is preferred to the commercial test sera or collected human serum as a control material.

Oberhalb einer bestimmten Analysenzahl ist im klinischchemischen Laboratorium eine tägliche Qualitätskontrolle unerläßlich $(1,2)$.

Da die hohe Zuwachsrate von Hepatitisfällen bei Laboratoriumspersonal $(3,4)$ Zurückhaltung in der Vetwendung menschlicher Sammelseren empfiehlt und die Verwendung käuflicher Testseren in größerem Umfang erhebliche Kosten verursacht, sind wir vor 8 Monaten zum Einsatz von Rinderserum in der Qualitätskontrolle von 16 klinisch-chemischen Routineanalysen übergegangen. Über erste Erfahrungen soll hier kurz berichtet werden.

\section{Methoden}

\section{Gewinnen von Rinderserum}

Frisches Rinderblut wird in einer Menge von etwa 10 Litern vom Schlachthof bezogen. Nach Eintritt der Gerinnung werden die zellulären Bestandteile abzentrifugiert. Eine geringe Hämolyse läßt sich nicht immer vermeiden. Die vereinigten Überstände werden sehr sorgfältig gemischt, um individuelle Unterschiede der einzelnen Fraktionen auszugleichen. In einigen Fällen (Bilirubin, Harnsäure), erfolgt Zusatz definierter Mengen der zu kontrollierenden Substanz. Das Serum wird anschließend in Portionen von etwa $2 \mathrm{ml}$ in verschließbare Polyäthylenröhrchen verteilt und bei $-20^{\circ}$ eingefroren.

\section{Arbeitsablauf}

$\mathrm{Zu}$ Beginn der Kontrollperiode werden für jede im Kontrollprogramm enthaltene Methode 20 Proben der verwendeten Rinderserumcharge auf einmal zur Analyse ausgegeben: Bestimmen des Mittelwertes $\bar{x}$ und der Präzision VK „,in Serie“. In der Folgezeit wird täglich für jede Methode eine neue Probe bei Raumtemperatur aufgetaut und ausgegeben. Die Assistentin, die das Kontrollserum herstellt und verteilt, sammelt täglich die Ergebnisse ein und registriert sie. Dieses Schema entspricht etwa dem von BürTrNeR (1) geforderten Minimalprogramm der Qualitätskontrolle.

\section{Analytische Methoden}

Die durchgeführten Analysenverfahren sind mit Literaturangaben (9-23) in Tabelle 1 aufgeführt. $\mathrm{Da}$ in dem von uns bisher verwendeten Rinderserum einige Komponenten praktisch nicht (z. B. Harnsäure) oder nur geringfügig (z. B. Bilirubin, Amylase) vorkommen, fügten wir $50 \mathrm{mg}$ Harnsäure sowie neuerdings $60 \mathrm{mg}$ Bilirubin (in 0,1 $\mathrm{N} \mathrm{Na}_{2} \mathrm{CO}_{3}$ gèlöst (7)) pro Liter Rinderserum in Reinsubstanz hinzu. Zur Amylasebestimmung verwendeten wir im Untersuchungsansatz das fünffache Probenvolumen.

\section{Ergebnisse und Diskussion}

Rinderserum eignet sich für die statistische Qualitätskontrolle einer Reihe von klinisch-chemischen Analysenmethoden, obwohl die Mittelwerte $\overline{\mathbf{x}}$ der einzelnen Bestandteile von Charge zu Charge etwas variieren. Tabelle 1 zeigt, welche Methoden wir bisher überprüft haben. In Tabelle 2 sind die von uns festgestellten Präzisionen der Verfahren aufgeführt und Vergleichsdaten von BüTTNER $(1,2,5)$, die sich auf einen sehr viel größeren Beobachtungszeitraum beziehen, und Ergebnisse eines Rundversuchs (24) eingetragen. Die Werte stellen das arithmetische Mittel der Präzision „von Tag zu Tag" dar.

Tab. 1

Verwendete Analysenverfahren

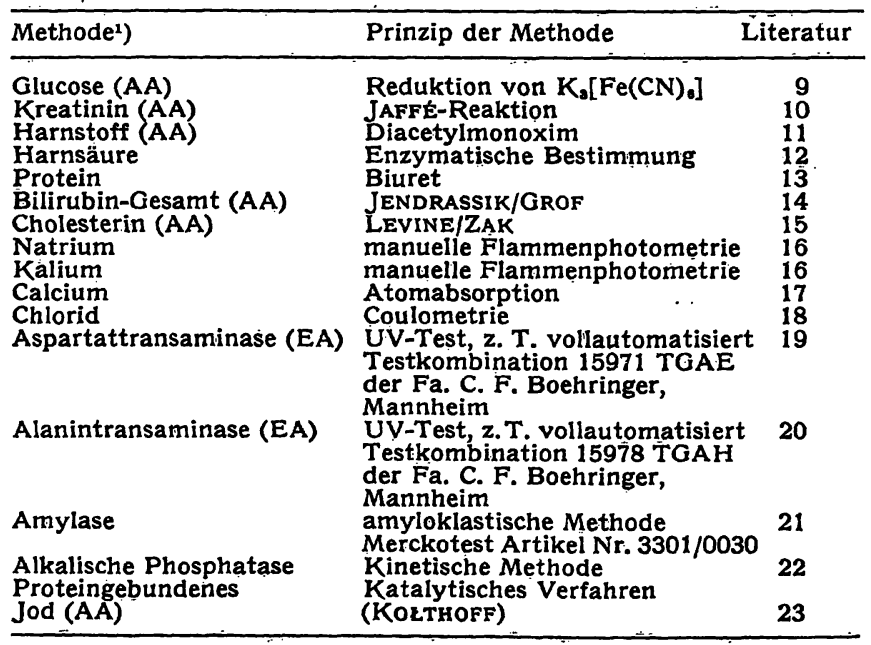

2) $\mathrm{AA}=$ Auto-Analyzer (Technicon)

$\mathrm{EA}=$ Enzym-Autómat (Eppendorf Gerätebau). 
Tab. 2

Präzision von Qualitătskontrollen mit Rinderserum

\begin{tabular}{|c|c|c|c|c|c|c|}
\hline \multirow[t]{2}{*}{ Methode') } & \multicolumn{2}{|l|}{ 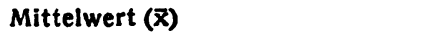 } & \multicolumn{4}{|c|}{ Präzision, VK(\%) } \\
\hline & Autoren & 1. c. (6) & $\underset{\text { Autoren }}{\text {,in Serie" }}(n=20)$ & Autoren & $\begin{array}{c}\text { "von Tag zu Tag'" } \\
\text { l. c. (1) }\end{array}$ & l. c. $\left.(24)^{\circ}\right)$ \\
\hline $\begin{array}{l}\text { Glucose (AA) } \\
\text { Kreatinin (AA) } \\
\text { Harnstoff } \\
\text { Harnsäure } \\
\text { Protein } \\
\text { Bilirubin-Gesamt (AA) }\end{array}$ & 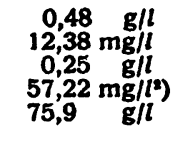 & & $\begin{array}{l}4,7 \\
1,3 \\
1,9 \\
9,7 \\
0,7\end{array}$ & $\begin{array}{l}3,6-4,6 \\
2,0-6,7 \\
2,0-7,0 \\
1,4-6,0\end{array}$ & $\begin{array}{l}2,5 \\
\frac{3,0}{1,6}\end{array}$ & $\begin{array}{l}4,8 \\
7,8 \\
7,8 \\
2,8\end{array}$ \\
\hline $\begin{array}{l}\text { Cholesterin (AA) } \\
\text { Natrium } \\
\text { Kalium } \\
\text { Calcium } \\
\text { Chlorid } \\
\text { Aspartattransaminase (EA) } \\
\text { Alanintransaminase (EA) } \\
\text { Amylase } \\
\text { Alkalische Phosphatase } \\
\text { Proteingebundenes Jod (AA) }\end{array}$ & $\begin{array}{rl}0,99 & \mathrm{~g} / \mathrm{l} \\
144,1 & \mathrm{mMol} / \mathrm{l} \\
6,25 & \mathrm{mMol} / \mathrm{l} \\
2,27 & \mathrm{mMol} / \mathrm{l} \\
95,2 & \mathrm{mMol} / \mathrm{l} \\
60,9 & \text { I E/l } \\
14,9 & \text { I } / l \\
32,2 & \text { I E } / l \\
150,4 & \text { I E } / l \\
71,0 & \mu \mathrm{g} / l\end{array}$ & $\begin{array}{r}152 \mathrm{mMol} / \mathrm{l} \\
5,9 \mathrm{mMol} / \mathrm{l} \\
2,7 \mathrm{mMol} / \mathrm{l} \\
92,8 \mathrm{mMl} / \mathrm{l}\end{array}$ & $\begin{array}{l}1,5 \\
2,6 \\
2,9 \\
2,6 \\
2,5 \\
4,4 \\
8,6 \\
9,3 \\
1,8 \\
2,3\end{array}$ & $\begin{array}{l}2,0-6,0 \\
1,3-5,0 \\
1,9-4,0 \\
3,6-7,7 \\
1,0-4,0 \\
3,7-7,0 \\
8,0-15,0 \\
15,0-20,0 \\
6,0-10,0 \\
2,58\end{array}$ & $\begin{array}{l}3,0 \\
1,4 \\
2,0 \\
3,0 \\
0,9 \\
6,0 \\
8,0 \\
\frac{7,0}{-}\end{array}$ & $\begin{array}{l}\overline{1,8} \\
3,2 \\
2,9 \\
2,2 \\
= \\
=\end{array}$ \\
\hline
\end{tabular}

1) s. Tab. $1 . \quad$ 2) nach Zusatz von $50 \mathrm{mg}$ Harnsăure/l.

o) noch keine längeren Erfahrungen.

9) Literatur (5). $\quad$ ") Bei Verwendung handelsüblicher Kontrollseren

Die Durchführung von Kontrollanalysen schon in der Aufbauphase unseres Zentrallaboratoriums ermöglichte es, den Einfluß organisatorischer Veränderungen und methodischer und apparativer Verbesserungen auf die Präzision der Ergebnisse zu kontrollieren. Die bisher erreichte Präzision ist $z w a r$ noch nicht zufriedenstellend. Wir erwarten aber eine zunehmende Steigerung der Genauigkeit durch wachsende Erfahrung und Sorgfalt des Laborpersonals im Umgang mit der Mechanik und Automatik der Analysengeräte. Die Kontrolle wird sich auf weitere Analysenmethoden ausdehnen lassen. Leider können mit diesem Untersuchungsmaterial keine Kontrollen bei Gerinnungsanalysen durchgeführt werden, da die meisten Gerinnungsfaktoren auch im tiefgefrorenen Zustand nicht haltbar sind.

Das preiswerte und gefahrlose Rinderserum wird von uns seit Jahren im klinisch-chemischen Unterricht von Medizinstudenten als Analysenmaterial verwendet.

Die Herstellung des Kontrollserums ist einfach und verursacht relativ wenig Aufwand an Zeit und Kosten. Als nachteilig ist die leichte Hämolyse anzusehen, die eine über dem Normalbereich von menschlichem Serum liegende Kaliumkonzentration bedingt.

Das Aufbewahren in der Tiefkühltruhe $\left(-20^{\circ}\right)$ ist sicher die bequemste Methode der Konservierung. Man vermeidet Volumenfehler beim Lösen von tiefgefrorenen Proben. Bei dieser Art der Konservierung haben sich die Konzentrationen von Natrium, Kalium, Chlorid, Calcium, Glucose, Harnstoff, Kreatinin, Gesamt-Protein und proteingebundenem Jod während einer Zeit von 6 Monaten nicht erkennbar verändert. Für die Haltbarkeit der Harnsāure bestehen noch wenig Anhaltspunkte, da wir die Methode èrst seit kurzem kontrollieren. Die Versuche mit der von uns beschriebenen Konservierungsmethode, Bilirubin im Rinderserum haltbar zu machen, waren bisher wenig befriedigend. Problematisch ist die Stabilität der Mehrzahl der Enzymaktivitäten. Die Aktivitāt der Amylase ist - allerdings bei sehr schlechter Präzision im unteren Normbereich wahrscheinlich stabil. Die Aktivität der alkalischen Phosphatase fiel regelmäßig um 5 bis $6 \%$ pro Monat ab. Eine Beurteilung des Verhaltens der Transaminasen, insbesondere die Interpretation des geringen Aktivitätsabfalles der Alanintransaminase ist noch nicht möglich, weil während des Beobachtungszeitraumes von einer manuellen auf eine vollautomatische Methode übergegangen wurde. Mit dem Analysenautomaten war in der Serie eine etwas bessere Präzision zu erreichen.

Die Zahl der jährlich gemeldeten und als Berufserkrankung anerkannten Fälle von Virushepatitis nimmt in der Bundesrepublik stark zu $(3,4)$. Neben dem Personal der internmedizinischen und Infektionsabteilungen ist besonders das Laborpersonal gefährdet. Solange die Identität des Hepatitisvirus und der Infektionsmodus nicht eindeutig geklärt sind, sollte der sogenannte Human-Serumpool als Infektionsquelle soweit als möglich ausgeschaltet werden (8), zumal auch anikterische Seren Hepatitisviren enthalten können. Über das Infektionsrisiko käuflicher Kontrollseren läßt sich bisher nichts Sicheres aussagen (8).

Es empfiehlt sich allerdings, Rinderserum auf Brucelloseinfektion zu prüfen. Die Komplementbindungsreaktion gegen Brucella abortus BANG fiel bisher bei uns stets negativ aus.

Nach Ansicht von Veterinärmedizinern ist das bei uns verwendete Material in dieser Hinsicht als unbedenklich anzusehen.

Wir danken unserem Institutskollegen Herrn Dr. J. S. BRAun für die Durchführung der serologischen Untersuchungen.

\section{Literatur}

1. BürTnER, H., diese Z. 5, 47 (1967). - 2. BüTTNER, H., diese Z. 7, 91 (1969). - 3. Jahresbericht über Unfallverhütung der Berufsgenossenschaft für Gesundheitsdienst und Wohlfahrtspflege. Hamburg, S. 14 (1968). - 4. KLAwIS, G. und F. EgGeling, Arbeitsmedizin, Sozialmedizin, Arbeitshygiene, 8295 (1967). -
5. Bürtrier, H. in: G. Griesser und G. Wagner (Hrsg.): Automatisierung des klinischen Laboratoriums. F. K. Schattauer Verlag, Stuttgart, New York (1968). - 6. Blood and other body fluids. Federation of American Societies for Experimental Biology, Washington, D. C. (1961). - 7. Standard Methods of Clinical 
Chemistry, Vol. V, S. 75 (1965): Recommendation on a uniform bilirubin standard. - 8. Arztemerkblatt: Ist die Hepatitis ein unvermeidbares Risiko? Deutsches Grünes Kreuz, Marburg/Lahn. 9. HoffmanN, W. S., J. Biol. Chemistry 120, 51 (1937) (Für den Auto-Analyzer modifiziert, Technicon-Vorschrift, N-9a). 10. Hawk, P. B., B. L. Oser und W. H. Sumimerson, Practical Physiological Chemistry, Philadelphia, Pa.: Blakiston Co. S. 506 (1954) (Für den Auto-Analyzer modifiziert, Technicon-Vorschrift, N-11 b). - 11. MARSH, W. H., B. Fingerhut und H. MTLLER, Clin. Chem. (New York) 11, 624 (1965) (Für den Auto-Analyzer modifiziert, Technicon-Vorschrift N-1b). - 12. ZölLNER, N., diese Z. 1, 178 (1963). - 13. Weichselbaum, T. E., Amer. J. Clin. Path. 10, 40 (1946). - 14. Jendrassik, L. und P. Grof, Biochem. Z. 297, 81 (1938) (Für den Auto-Analyzer modifiziert, Technicon-Vorschrift N-12a). - 15. LEvine, J. und B. ZAK, Clin. chim. Acta (Amsterdam) 10, 381 (1964) (Für den Auto-Analyzer modifiziert, Technicon-Vorschrift N-37a). - 16. Handbuch Photometer Eppendorf, II. Aufl., Netheler u. Hinz GmbH, Hbg. (1965). - 17. WrLlis, J. B., Spectrochim. Acta (Ĺondon) 16, 259 (1960) und Handbuch der Fa. Perkin-Elmer zum Atomabsorptionsspektrophotometer 303. - 18. Cotrove, E., H. V. TRANTham und R. L. Bowman, J. Laborat. Clin. Med. S. Louis 51, 461 (1958). - 19. Karmen, A., J. Clin. Invest. 34, 131 (1955). 20. Wroblewski, F. und J. S. LaDue, Proc. Soc. Exp. Biol. Med. 91, 569 (1956). - 21. Street, H. V. und J. R. Crose, Clin. chim. Acta Amsterdam 1, 256 (1956). - 22. Hausamen, T. U., R. HeLGER, W. Rick und W. Gross, Clin. ćhim. Acta Amsterdam 15, 241 (1967). - 23. Kolthoff, J. M. und E. B. Sandele, J. Amer. chem. Soc. 56, 1426 (1934) (Für den Auto-Analyzer modifiziert. Technicon-Instruction Manual No. PB/D 1). - 24. STAMM, D, und H. BürtrNer, diese Z. 7, 393 (1969).

Dr. K. Borner

1 Berlin 45, Hindenburgdamm 30

Klinikum d. FU Berlin 